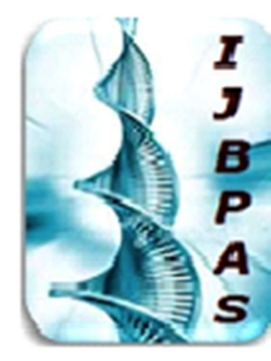

International Journal of Biology, Pharmacy and Allied Seiences (IJBPAS) 'A Bridge Betusen Caboratory and QRendo'

\author{
www.ibpas.com
}

\title{
REVIEW: TRANSDERMAL PATCH
}

\section{RAVI V. PATEL, GAZALA Y. ANSARI, SURAJ R. CHAUHAN AND JITENDRA O. BHANGALE*}

Smt. N. M. Padalia Pharmacy College, Sarkhej Changodar Road, Navapura, Ahmedabad-382210, Gujarat, India

*Corresponding Author: E Mail: Jitendra O. Bhangale: џitu2586@gmail.com Received $9^{\text {th }}$ May 2021; Revised 10 ${ }^{\text {th }}$ July 2021; Accepted 29 ${ }^{\text {th }}$ Aug. 2021; Available online $15^{\text {th }}$ Dec. 2021 https://doi.org/10.31032/IJBPAS/2021/10.12.1010

\begin{abstract}
Transdermal route is very easy administration of drug to people offers to relatively painless. Transdermal drug delivery was first used in 1981that drugs which applied to through the skin provide systematic effect. The patch provided a controlled release of the medication into the patient, usually through either a porous membrane covering a reservoir of medication or through body heat melting thin layers of medication embedded in the adhesive. This is followed by consideration of factors, mechanism, preparations, various patch designs and evaluation to be used for transdermal drug delivery system. In today's scenario there are many issues associated with People safety and regulatory aspects with the use of currently marketed products. The review concluded by examining future progress of transdermal patches and drug delivery systems, various approaches, conditions and methodologies.
\end{abstract}

Keywords: Transdermal drug delivery system, transdermal patch

\section{INTRODUCTION}

A transdermal patch is a medicated adhesive patch which is used on the skin for delivering the drug in a specified dose in a particular region through skin. The main advantage of the transdermal drug delivery system over the other route of administrations is its controlled release of the drug through skin usually by a porous membrane covering the 
medication or through body temperature which melts down the thin layers of medication embedded in the adhesive. Currently Medication like oscine, nicotine, estrogen, vasodilator and topical anesthetic were administered using patches. Now a days demand on Non-medicated patches are high like thermal-cold patch, nutrient patch, skin care patch, aroma patch etc. So, thetransdermal drug delivery system has more suitable than conventional drug delivery system. In transdermal drug delivery system, Drugs can penetrate through different pathways like hair follicles, sebaceous glands and sweat duct. Transdermal drug delivery system (TDDS) is a discrete dosage forms of patches, topically administered, drug reaches to the systemic circulation via skin at a predetermined and controlled rate over a prolonged time resulting in improvement of therapeutic efficacy with lesser side effect of drug. TDDS maintains drug concentration within the therapeutic window for prolong period of time ensuring that drug levels neither fall below the minimum effective concentration nor exceed the maximum effective concentration [1-6].

ADVANTAGES [7]
- The transdermal drug provides a continuous infusion of a drug over a long period of time.

- An equivalent therapeutic effect can be produced by transdermal medication at a lower dose than necessary.

- Self-administration is possible.

- In emergencies, they can be quickly and easily identified on the basis of their physical presence, characteristics and distinctive features.

- Can be used for drugs with a narrow therapeutic window.

- A long duration of action reduces the frequency of dosing.

- Greater comfort in administering medications.

- Improved bioavailability.

- More uniform plasma levels and maintenance of plasma concentration of effective drugs.

- Reduced side effects and improved therapy by maintaining plasma levels until the end of the dosing interval.

- Flexibility to stop drug delivery simply by removing the skin patch.

- Better compliance and patient comfort through easy, non-invasive and painless application.

- Avoid variations between and within the patient 


\section{DISADVANTAGES [8]}

- Many drugs, especially drugs with a hydrophilic structure, penetrate the skin too slowly to have a therapeutic effect.

- The barrier function of the skin changes from one place to another in the same person, from one person to another and also with age.

- Currently, only small lipophilic drugs can be delivered through the skin.

- The drug molecule must be potent because the size of the patch limits the amount that can be delivered.

- Not suitable for high doses of medication.

- Adhesion may vary depending on the plaster type and environmental conditions.

- Skin irritation and hypersensitivity reactions may occur.

- Medicines that require high blood levels cannot be given.

- In addition to these limitations, the high cost of the product is also a major drawback of the wide.

\section{PRINCIPLE OF TRANSDERMAL PERMEATION}

Previously, the skin was considered an impenetrable protective barrier, but further studies were conducted demonstrating the usefulness of the skin as a systemic route of administration. The skin is the most intense and easily accessible organ in the body, as only a fraction of a millimeter of tissue separates its surface from the underlying capillary network. The different steps involved in the transport of the drug from the patch to the systemic circulation are as follows: Medication delivery from the medication reservoir to the flow control membrane, diffusion of the drug from the membrane that limits the rate to the stratum corneum, sorption through the stratum corneum and penetration through the viable epidermis, uptake of the drug through the capillary network in the dermal papillary layer and effect on the target organ.

\section{ANATOMY AND PHYSIOLOGY OF SKIN}

Human skin comprises of three distinct but mutually dependent tissues: Epidermis: The multilayered epidermis varies in thickness depending on the cell size and number of cell layers in the epidermis, ranging from $0.8 \mathrm{~mm}$ on the palms and soles to $0.06 \mathrm{~mm}$ on the palms. It consists of the outer stratum corneum and the viable epidermis. Stratum corneum: It is the outermost layer of the skin, also called the stratum corneum. It is about $10 \mu \mathrm{m}$ thick when dry, but swells to several times that thickness when fully hydrated. It contains 10 to 25 layers of dead 
cornified cells called corneocytes. It is flexible but relatively waterproof. The stratum corneum is the main barrier to drug penetration. The architecture of the stratum corneum can be modeled as a wall-like structure. In this model, the cornified cells function as protein "bricks" integrated into a lipid "mortar". Lipids are arranged in several bilayers. There is sufficient amphiphilic material in the lipid fraction, such as polar free fatty acids and cholesterol, to maintain a bilayer shape. Viable epidermis: It is located below the stratum corneum and ranges in thickness from $0.06 \mathrm{~mm}$ on the eyelids to 0.8 $\mathrm{mm}$ on the palms. Going inward, it consists of several layers such as stratum lucidum, stratum granulosum, stratum spinosum and stratum basal. In the basal layer, cell mitosis constantly renews the epidermis and this proliferation compensates for the loss of dead cells from the skin surface. As the cells produced by the basal layer move outward, they change morphologically and histochemically, undergoing keratinization to form the outermost layer of the stratum corneum.

Dermis: The dermis is a layer 3-5 mm thick and consists of a matrix of connective tissue, which contains blood vessels, lymphatic vessels and nerves. The blood supply to the skin has an essential function in regulating body temperature. It also supplies nutrients and oxygen to the skin while removing toxins and waste. The capillaries extend up to $0.2 \mathrm{~mm}$ from the skin's surface and provide dripping conditions for most molecules that penetrate the skin barrier. Thus, the blood supply keeps the skin concentration of a permeant very low and the resulting difference in concentration across the epidermis provides the concentration gradient essential for transdermal permeation. Hypodermis: The dermis or subcutaneous fatty tissue supports the dermis and epidermis. It serves as a fat storage area. This layer provides temperature regulation, nutritional support, and mechanical protection. It carries major blood vessels and nerves to the skin and may contain pressuresensing organs. For transdermal drug use, the drug must penetrate through these 3 layers and reach the circulatory system, while in the case of topical application, the drug only penetrates through the stratum corneum mainly and then is stored. drugs in the layers of the skin. [9].

\section{FACTORS (PHYSICOCHEMICAL} PROPERTIES OF PERMEATE) [10-11]

Partition coefficient: Water-soluble and fatsoluble drugs are favorably absorbed through the skin. The intercellular pathway is applicable for drugs with an intermediate 
partition coefficient ( $\log \mathrm{K} 1$ to 3$)$ and with a high lipophilicity. The transcellular pathway probably predominates for more hydrophilic molecules $(\log \mathrm{K}<1)$.

Molecular size: There is an inverse relationship between transdermal flux and the molecular weight of the molecule. The drug molecule selected as candidates for transdermal administration tends to fall within a narrow molecular weight range. (100-500 Dalton).

Solubility/melting point: Lipophilicity is a desirable property of transdermal candidates because lipophilic molecules tend to pass through the skin faster than more hydrophilic molecules. High melting point drugs have relatively low solubility in water at normal temperature and pressure.

Ionization: According to $\mathrm{pH}$-partition hypothesis, only the unionized form of the drug can permeate through the lipid barrier in significant amounts.

FACTORS (PHYSIOLOGICAL \& PATHOLOGICAL CONDITIONS OF SKIN)

Reservoir effect of horny layer: The reservoir effect of horny layer which is deeper layer is due to irreversible binding of a part of the applied drug with the skin.

Lipid film: The lipid film on the skin surface acts as a protective layer to prevent the removal of moisture from the skin and helps in maintaining the barrier function of stratum corneum.

Skin hydration: Skin hydration can be achieved simply by covering or occluding the skin with plastic sheeting, leading to accumulation of sweat \& enhance the penetration by opening the densed, closely packed cells of the skin and increase its porosity.

Skin temperature: Increase in skin temperature increases the rate of skin permeation this is due to availability of energy required for diffusivity.

Regional variation: Differences in nature and thickness of the barrier of skin cause variation in permeability.

Pathological injuries to the skin: Injuries that disrupt the continuity of the stratum corneum, increases permeability due to increased vasodilatation caused by removal of the barrier layer.

Cutaneous self-metabolism: Catabolic enzymes present in the epidermis may render the drug inactive by metabolism and thus the topical bioavailability of the drug.

Skin barrier properties in the neonate and young infant: The $\mathrm{pH}$ of the skin surface of newborns is higher than that of the skin of adults. The surface of the skin of newborns is slightly hydrophobic and relatively dry and 
rough compared to that of older infants. The hydration of the stratum corneum stabilizes around 3 months of age.

Skin barrier properties in aged skin: There are changes in the physiology of aging skin (> 65 years old). Corneocytes have been shown to increase in surface area, which may affect stratum corneum function due to the resulting decrease in the volume of the intercorneocyte space per unit volume of the stratum corneum. The moisture content of human skin decreases with age.

Race: Racial differences between blacks and whites have been shown in several anatomical and physiological functions of the skin, although data are relatively scarce. In blacks, increased intracellular association, higher lipid content and a higher degree of skin electrical resistance than whites have been demonstrated.

Body site: The structure of the skin varies in different places in the body. Genital tissue generally provides the most permeable site for transdermal drug delivery. The skin of the head and neck is also relatively permeable compared to other places on the body, such as the arms and legs. The intermediate permeability for most drugs is found on the trunk of the body.

Penetration enhancers used: The low permeability of drugs through the skin can be improved through the development of penetration enhancers. Penetration enhancers or promoters are agents that do not have inherent therapeutic properties, but can transport drug sorption from drug delivery systems to the skin and/or their subsequent transdermal permeation through the skin.

\section{APPROACHES FOR DEVELOPING TRANSDERMAL DRUG DELIVERY SYSTEM [12-16]}

There are four different approaches that have been utilized to obtain Transdermal Drug Delivery Systems

\section{Membrane permeation controlled systems:} In this TDDS, the drug reservoir is completely encapsulated in a shallow compartment mounted on a drugimpermeable metal-plastic laminate and a drug-control membrane. speed composed of polymers that may be microporous or nonporous e.g. Ethylene Vinyl Acetate copolymer (EVA), with a defined drug permeability property. The main advantage of this transdermal system is the constant delivery of the drug. e.g. Transdermal system that releases nitroglycerin.

Adhesive dispersion type systems: This TDDS is similar to the membrane permeation controlled system, but simpler than that. The drug is dispersed directly onto the adhesive polymer and this adhesive is spread over the 
solvent-impermeable or hot-melt thin metallic plastic laminate, which acts as a drug reservoir. e.g. Isosorbide dinitrate Transdermal Therapeutic System.

Matrix diffusion-controlled systems: In this drug is dispersed on the hydrophilic or lipophilic polymer matrix. This drug matrix is carefully transferred to a plastic drug disk with a predefined area and thickness. This drug-containing polymeric disc is then transferred to a fabricated waterproof plastic carrier. The adhesive polymer is then placed around this disc to form the adhesive edge.

Micro reservoir type or micro sealed dissolution: This is a fusion model of a trolled system: both reservoir and matrix type of TDDS. First, the drug solids are suspended in an aqueous solution of the water-soluble liquid polymer and this is redispersed in a lipophilic polymer to form several discrete microscopic spheres that release drug reservoirs.

\section{METHODS OF PREPARATION OF TDDS [17-18]}

\section{Asymmetric TPX Membrane Method:}

This method was discovered in 1994 by Berner and John. With this method, a prototype plaster can be manufactured using a heat-sealable polyester film (type 1009.3 $\mathrm{m})$ with a concave diameter of $1 \mathrm{~cm}$ as a support membrane. Drug dispersed on a concave membrane, covered with an asymmetric TPX [poly(4methyl1 pentene)] membrane and sealed with an adhesive. They are prepared using either a dry or wet reverse process. In this TPX is dissolved in a mixture of solvents (cyclohexane) and solvent-free additives at $60{ }^{\circ} \mathrm{C}$ to form a polymer solution. The polymer solution was maintained at $40{ }^{\circ} \mathrm{C}$ for $24 \mathrm{~h}$ and poured onto a glass dish. Then evaporate the cast film at $50{ }^{\circ} \mathrm{C}$ for $30 \mathrm{~s}$, then immediately immerse the glass plate in the coagulation bath (keeping temperature at $25^{\circ} \mathrm{C}$ ). After soaking for $10 \mathrm{~min}$, the film can be removed, air-dried in a recirculating oven at $50{ }^{\circ} \mathrm{C}$ for $12 \mathrm{~h}$.

Circular Teflon Mould Method: It was discovered in 1989 by Baker and Heller. As the organic solvent, a polymer solution is used in different proportions. Then this solution is divided into two parts. In one part, the calculated amount of the drug is dissolved, and in another part, enhancers of different concentrations are dissolved, and then two parts are mixed. Next, the plasticizer (e.g., diNbutyl phthalate) is added to the drug polymer solution. The total contents should be stirred for 12 hours and then poured into a circular Teflon mold. The mold should be placed on a flat surface and covered with an inverted funnel to control solvent evaporation in a laminar flow hood 
model with an air velocity of $0.5 \mathrm{~m} / \mathrm{s}$. The solvent is allowed to evaporate for 24 hours. Thereafter, a dried film has formed which must be kept for an additional 24 hours at 25 $\pm 0.5{ }^{\circ} \mathrm{C}$ in a silica gel desiccator before evaluation to eliminate the effects.

Mercury Substrate Method: In this method, the drug and plasticizer are dissolved in a polymer solution. It is stirred for 10 to 15 minutes to produce a homogeneous dispersion, then it is poured onto a flat mercury surface, covered with an inverted funnel to control the evaporation of the solvent.

"IPM Membranes" Method: In the mixture of water and polymer (propylene glycol polymer containing Carbomer 940), the drug is dispersed and stirred in a magnetic stirrer for 12 hours. The dispersion must be neutralized and made viscous by adding triethanolamine. If the solubility of the drug in aqueous solution is very low, the gel is obtained in solution using a buffer $\mathrm{pH}$ 7.4. The gel formed is incorporated into the IPM membrane.

"EVAC Membranes" Method: For the preparation of TDS, a membrane of 1rbopol reservoir gel, polyethylene (PE) and Ethylene Vinyl Acetate Copolymer (EVAC) is required as the flow control membrane. If the drug is insoluble in water, use propylene glycol for the preparation of the gel. The drug is dissolved in propylene glycol, carbopol resin is added to the above solution and neutralized with $5 \% \quad \mathrm{w} / \mathrm{w}$ sodium hydroxide solution. The drug (in the form of a gel) is placed on a sheet of the carrier layer covering the specified area. A flow control membrane will be placed over the gel and the edges will be sealed to obtain a watertight device.

Proliposomes: By an assisted method using membrane deposition technique, proliposomes were prepared. The drug/lecithin ratio should be $0.1: 2.0$ to be considered as the optimal ratio compared to previous references. To prepare proliosomes in a $100 \mathrm{ml}$ round bottom flask, take $5 \mathrm{mg}$ of mannitol powder, then maintain at $6070{ }^{\circ} \mathrm{C}$ and rotate the flaskat $8090 \mathrm{rpm}$ andthe mannitol is dried under vacuum for $30 \mathrm{~min}$. After drying, the temperature of the water bath was adjusted to $20-30{ }^{\circ} \mathrm{C}$. The drug and lecithin were dissolved in a suitable mixture of organic solvents, an amount of $0.5 \mathrm{ml}$ of the organic solution was added. round bottom flask at $37{ }^{\circ} \mathrm{C}$, after complete drying, a second portion $(0.5 \mathrm{ml})$ ofsolution should be added. After the final charge, the vial containing the proliposomes is placed in a freeze dryer and then drug loaded mannitol powders (proliposomes) are placed in a 
desiccator overnight and then sieved through 100 mesh. The collected powder is transferred to a glass vial and stored at freezing temperature until characterization.

Free Film Method: In this process, a cellulose acetate-free film is first made by casting it on a mercury surface. And with chloroform, a $2 \% \mathrm{w} / \mathrm{w}$ polymer solution is prepared. Plasticizers should be added at a concentration of $40 \% \mathrm{w} / \mathrm{w}$ polymer weight. Then $5 \mathrm{ml}$ of polymer solution is poured into a glass ring which is placed on the mercury surface in a Petridis glass. The solvent evaporation rate can be controlled by placing an inverted funnel on the Petridis. The formation of the film is observed by observing the surface of the mercury after complete evaporation of the solvent. The dry film is separated and stored between sheets of wax paper in a desiccator until use. This process allows us to make free films of different thicknesses that can be prepared by changing the volume of the polymer solution.

\section{COMPONENTS OF TRANSDERMAL}

\section{DRUG DELIVERY SYSTEM [19-23]}

Polymer matrix/Drug reservoir: Polymers are an integral part of TDDS. It is prepared by dispersing the drug in a liquid or solid synthetic polymer base. The polymers (Table 1) used in TDDS must have biocompatibility and chemical compatibility with the drug and other system components such as penetration enhancers. In addition, they must ensure consistent and effective drug delivery over the expected shelf life of the product and must be safe.

Table 1: Different Types of Polymers Used in TDDS

\begin{tabular}{|c|c|c|}
\hline Natural Polymers & Synthetic Elastomers & Synthetic Polymers \\
\hline Cellulose derivatives, & $\begin{array}{c}\text { Polybutadiene, Hydrin rubber, } \\
\text { polysiloxane, silicone rubber, }\end{array}$ & $\begin{array}{c}\text { Polyethylene, Polypropylene, } \\
\text { Polyacrylate, Polyamide, } \\
\text { Pein, Gelatin, Waxes, Proteins, } \\
\text { Gums, Natural rubber, Starch. }\end{array}$ \\
$\begin{array}{c}\text { Nitrile, Acrylonitrile, } \\
\text { Butylrubber, Styrenebutadiene, } \\
\text { Neoprene etc. }\end{array}$ & $\begin{array}{c}\text { Polymethyl methacrylate, } \\
\text { Epoxy, Polyurea, etc. }\end{array}$ \\
\hline
\end{tabular}

Drug: The drug is in direct contact with the non-stick coating. Example: nicotine, methotrexate and estrogen. Some of the desirable properties of a drug for transdermal delivery are the following: The drug molecule must be sufficiently soluble in oil and water, The drug should have a molecular weight of less than about 1000 Daltons, The drug must have a low melting point and The drug molecule will need a balanced partition coefficient to penetrate the stratum corneum Permeation enhancers: Chemical compounds that increase the permeability of the stratum corneum to achieve higher therapeutic levels of the drug candidate. They improve permeability by interacting with 
structural components of the stratum corneum. Ideal Properties of Permeation Activators: They should be non-irritating, non-toxic and non-allergic, they must not bind to the receptor site, ie show no pharmacological activity and they should be cosmetically acceptable and feel good on the skin.

Pressure Sensitive Adhesive (PSA): The pressure sensitive adhesive helps the transdermal patch adhere to the skin surface. It can be easily removed from the smooth surface without leaving any residue. Adhesives based on polyacrylate, polyisobutylene and silicone are widely used in TDDS. Backing laminate: Supporting laminates are a carrier material that is impermeable to drugs and also to permeation enhancers. They must be chemically compatible with the drug, activator, adhesive, and other excipients. Vinyl, polyethylene and polyester films. Release liner: The non-stick coating is the main packaging material that can protect the patch, which will peel off when the patch is applied to the skin. The non-stick coating consists of a base layer which can be non-occlusive (eg tissue paper) or occlusive (eg polyethylene, polyvinyl chloride) and a non-stick layer composed of silicone or Teflon. The release liner must be chemically inert and must be permeable to drugs, penetration enhancers and water.

Other excipients like plasticizers and solvents: The solvents used are chloroform, methanol, acetone, isopropanol and dichloromethane. Plasticizers (Table 2) used dibutyl phthalate, triethyl citrate, polyethylene glycol and propylene glycol.

Table 2: Plasticizers Used in Transdermal Films

\begin{tabular}{|c|c|c|}
\hline Group & Hydrophilic/ Lipophilic & Plasticizer \\
\hline Glycerol and esters & Hydrophilic & Glycerine, Glycerine triacetate, Glyceryl tributyrate \\
\hline Glycol derivatives & Hydrophilic & Propylene glycol, Polyethylene glycol \\
\hline Phthalic acid esters & Lipophilic & Diethyl phthalate, Dibutyl phthalate \\
\hline Sebacic acid esters & Lipophilic & Diethyl sebacate, Dibutylsebacate \\
\hline Oleic acid esters & Hydrophilic & Oleiloleate \\
\hline Sugar alcohols & Hydrophilic & Sorbitol \\
\hline Citric acid esters & Hydrophilic & Triethyl citrate, Tributyl citrate \\
\hline Tartaric acid esters & Lipophilic & Diethyl tatarate \\
\hline
\end{tabular}

MECHANISM OF ACTION OF Iontophoresis: Iontophoresis causes a few

\section{TRANSDERMAL PATCH [24-25]}

The application of the transdermal patch and the flow of the active drug constituent from the patch to the circulatory system via skin occur through various methods. milliamps of current to pass a few square inches of skin through the electrode in contact with the formulation, facilitating drug delivery across the barrier. Mainly used for the administration of pilocarpine to induce 
sweating as part of the diagnostic test for cystic fibrosis. Iontophoretic administration of lidocaine appears to be a promising approach for rapid onset of anesthesia.

Electroporation: Electroporation is a method of applying short, high voltage electrical pulses to the skin. After electroporation, the permeability of the skin to diffusion of drugs is increased by 4 orders of magnitude. It is believed that the electrical pulses form temporary aqueous pores in the stratum corneum, causing drug transport. It is safe and the electrical impulses can be delivered painlessly using electrodes placed close together to strain the electrical field in the nerveless stratum corneum.

Application by ultrasound: The application of ultrasound, especially low-frequency ultrasound, has been shown to improve the transdermal transport of various drugs, including macromolecules. It is also known as sonophoresis. Katz et al. reported the use of low-frequency sonophoresis for topical application of EMLA cream.

Use of microscopic projection: Transdermal patches with microscopic projections called micro needles were used to facilitate transdermal drug transport. Needles ranging from approximately $10100 \mu \mathrm{m}$ in length are arranged in arrays. When pressed into the skin, the arrays make microscopic punctures that are large enough to deliver macromolecules, but small enough that the patient does not feel the penetration or pain. The drug is surface coated on the microneedles to aid in rapid absorption. They are used in development of cutaneous vaccines for tetanus and influenza. Various other methods are also used to apply transdermal patches such as thermal, electromagnetic and photomechanical poration. However, these methods are in the early stages of development and need further research.

\section{TYPES OF TRANSDERMAL PATCH} [26]

Single-layer Drug-in-Adhesive: The adhesive layer of this system also contains the drug. In this type of patch, the glue layer is not only responsible for bonding the different layers together, as well as the entire system to the skin, but also responsible for releasing the drug. The adhesive layer is surrounded by a temporary primer and backing.

Multi-layer Drug-in-Adhesive: The multilayer medicated adhesive plaster is similar to the single-layer system in that the two adhesive layers are also responsible for the release of the drug. However, the multi-layer system is different in that it adds an extra layer of medicinal adhesive, usually 
separated by a membrane (but not always). This patch also has a temporary liner and permanent support.

Reservoir: Unlike Drugin's single and multilayer adhesive systems, the transdermal reservoir system has a separate drug layer. The drug layer is a liquid compartment containing a drug solution or suspension separated by the adhesive layer. This patch is also supported by the backing layer. In this type of system, the release rate is zero order.

Matrix: The Matrix System has a drug layer of a semi-solid matrix containing a drug solution or suspension. The adhesive layer of this patch surrounds the drug layer that partially covers it.

Vapour Patch: With this type of plaster, the adhesive layer is not only used to bond the different layers together, but also to release the steam. Steam plasters are new to the market and release essential oils for up to 6 hours. The vapor patches release essential oils and are mainly used for unblocking. Other vapor patches on the market are controller vapor patches that improve sleep quality. There are also vapor patches on the market that reduce the amount of cigarettes smoked per month.

EVALUATION OF TRANSDERMAL DRUG DELIVERY SYSTEM [27-29]
Interaction Studies: To produce a stable product, the drug and the excipient must be compatible with each other. The drugexcipient interaction will affect the stability and bioavailability of the final formulation. Under that condition, if the excipients are new, they must first be used with the active ingredient in the formulation. The study of compatibility or interaction is very important. Interaction studies are performed by thermal analysis, Fourier transform infrared spectroscopy (FTIR), ultraviolet (UV) and chromatographic techniques by comparing their physicochemical properties such as dosage, melting point, number of waves and maximum absorbance.

Thickness of the patch: At various points, the thickness of the patch is measured with a digital micrometer and determines its average thickness and standard deviation.

Weight of uniformity: Before testing, the patch is dried at $60^{\circ} \mathrm{C}$ for 4 hours. Cut this patch into several parts and weigh in a digital scale. Take the average weight and calculate the standard deviation of the individual weight.

Folding endurance: A strip is cut with a specific area. Fold this strip over and over at some point until it breaks. The number of breaks in the strip film gives the value of the bending durability. 
Percentage moisture content: Patch or film is weighed first then it is kept in desiccator containing calcium chloride at room temperature. Taken it out after $24 \mathrm{hrs}$ again reweighed \& percentage moisture content is calculated by following formula:

Percentage moisture content $(\%)=$ $\frac{\text { Initial weight }- \text { Final weight }}{\text { Final weight }} \times 100$

Percentage moisture uptake: The patch was weighed separately and then stored in a desiccator containing a saturated solution of potassium chloride to maintain $84 \%$ Rhesus coefficient (RF), after which the membrane was reweighed and the percentage hygroscopicity was calculated as following formula.

Percentage moisture content (\%) = $\frac{\text { Initial weight }- \text { Final weight }}{\text { initialweight }} \times \mathbf{1 0 0}$

\section{Water vapor permeability (WVP)} evaluation: The WVP can be determined by the following formula:

$$
\mathrm{WVP}=\frac{\mathrm{W}}{\mathrm{A}}
$$

Where, WVP is expressed in $g / \mathrm{m}^{2}$ per $24 \mathrm{~h}$,

$W$ is the amount of vapor permeated through the patch expressed in $\mathrm{g} / 24 \mathrm{~h}$

$A$ is the surface area of the exposure samples expressed in $\mathbf{m}^{2}$.

Drug content: Take the patch with a specific area, dissolve it in a specific volume of solvent. The solution is then filtered and the drug content is analyzed by the appropriate method (UV or HPLC technique). Then take the average of three different samples.

Uniformity of dosage unit test: Take ten patches and the contents determined for the individual patches. If 9 out of 10 patches have a content between 85 and $115 \%$ of the specified value and one patch has a content of at least 75 to $125 \%$ of the specified value, then the transdermal patches pass the consistency test. But if 3 patches have a content between 75 and $125 \%$, then another 20 patches are tested for drug content. If these 20 patches have a range of 85 to $115 \%$, then the transdermal patches pass the test.

Polaroscope evaluation: This examination determines, using a polaroscope, whether the drug is present in the final formulation in amorphous or crystalline form. A surface area patch is held on the 1-object slide and observed for drug crystals.

Shear adhesion test: This test determines the cohesive strength of the adhesive polymer. The influencing factors are the type and composition of the polymers, their molecular weight, the degree of cross-linking and the amount of tackifier added. A coated adhesive tape is applied to a stainless steel plate, a certain weight is hung on the tape to affect it by pulling in the direction parallel to the plate. The shear adhesion test is 
determined by measuring the time it takes to remove the tape from the plate. The longer the pull-back time, the higher the shear strength.

Peel adhesion test: Here, the peel adhesion is the force required to remove an adhesive coating from a substrate. A single tape is applied to a stainless steel plate, then the tape is pulled at a $180^{\circ}$ angle from the substrate and the amount required to pull the tape is measured.

Thumb tack test: This test determines the adhesive property of the adhesive. The thumb is pressed on the glue and the adhesion is determined.

Flatness test: Three length strips are cut from different parts of the foil. The length of each strip measured and the variation in length due to non-uniformity of flatness are measured by determining the percent narrowing, where $0 \%$ narrowing equals $100 \%$ flatness.

\section{Percentage elongation break test:}

Percentage elongation can be determined by using following formula:

Elongation percentage $=$

$$
\frac{\mathrm{L} 1-\mathrm{L} 2}{\mathrm{~L} 2} \times 100
$$

Where $L_{1}$ is the final length of each strip $L_{2}$ is the initial length of each strip.

Rolling ball tack test: This test determines the smoothness of the polymer covering the floor. Here, the 7/16 inch diameter stainless steel ball is released onto an inclined track so that it rolls down and contacts the upward facing horizontal adhesive. The distance the ball travels along the glue track gives the tack measurement expressed in inches.

Quick stick (peel tack) test: Here, the tape is peeled off the substrate at $90^{\circ} \mathrm{C}$ at a rate of 12 inches / $\min$. The peel force required to break the bond between the adhesive and the substrate is measured and recorded as the tack value, which is expressed in ounces or grams per inch of width.

Probe tack test: Here the probe with the specific surface is kept in contact with the adhesive to form a connection between them. The probe is then removed to break it mechanically. The force required to pull the probe is the adhesion measured in grams.

In vitro drug release studies: The paddleover-disk method (USP apparatus V) can be used to evaluate the release of active ingredient from the patches. Here the film with a defined thickness is shaped, weighed, fixed on a glass plate with glue. It is stored in $500 \mathrm{ml}$ of phosphate buffer $(\mathrm{pH} \mathrm{7.4)}$ as the dissolution medium and the device is adjusted to $32 \pm 0.5^{\circ} \mathrm{C}$. Hold the paddle one inch from the glass plate and run it at a speed of 50 RPM. Withdraw $5 \mathrm{ml}$ from the sample canister at specified time intervals over 24 
hours and analyze by UV or HPLC. Perform the experiment in triplicate.

In vitro skin permeation studies: $A n$ in vitro skin permeation study is performed using diffusion cells. Male Wistar rats weighing 200-250 $\mathrm{g}$ are used here. Take the abdominal skin of the rat by removing the hair from the abdominal region with an electric razor. Then, the skin side of the skin is washed with distilled water to remove adhering tissue, and then kept in solution medium or phosphate buffer $\mathrm{pH} 7.4$ for 1 hour. Before the start of the experiment, it was placed on a magnetic stirrer with a small magnetic needle to evenly distribute the diffusion agent. The cell temperature was maintained at $32 \pm 0.5 \circ \mathrm{C}$ using a thermostatically controlled heater. Rat skin is placed between the compartments of the diffusion cell with the epidermis facing up in the donor compartment. A certain volume is removed from the receptor compartment at specified time intervals and the same volume of fresh sample is added. The sample is filtered and analyzed by UV or HPLC. The flow can be determined by plotting the slope between the steady state values of the amount of drug permeated in $\mathrm{mg} \mathrm{cm} 2$ versus time in hours and the permeability coefficient was obtained by dividing the flow by the initial drug load in $\mathrm{mg} \mathrm{cm}^{2}$.
Skin irritation test: This study was carried out in healthy rabbits (mean weight 1.21.5 $\mathrm{kg}$ ). Remove the surface of the rabbit's back by shaving it and wipe it clean with rubbing alcohol. The formulation is applied to the surface of the skin and is removed after 24 hours. The skin should be observed and classified into 5 levels according to the severity of the skin lesion.

Stability studies: Stability studies were performed according to ICH guidelines, with TDS samples stored at $40 \pm 0.5^{\circ} \mathrm{C}$ and $75 \pm$ $5 \% \mathrm{RH}$ for 6 months. Samples were taken after $0,30,60,90$ and 180 days and were suitably analyzed for active ingredient content.

\section{CONCLUSION}

The transdermal drug delivery system is a targeted delivery system where drug acts on the targeted site. TDDs mainly help to maintain the health of skin by preventive or therapeutic approaches through different mechanism. TDDS act as a transdermal patch, nano emulsion, nano suspension, containing novel carrier to enhance bioavailability which reduce the infection on the skin. Through exhaustive study this review gives basic and recent information regarding skin components, mechanism, advantages of TDDS, approaches and evaluation parameters of TDDS. 


\section{CONFLICT OF INTEREST:}

The authors declare that there is no conflict of interests.

\section{REFERENCES}

[1] Saroha K, Yadav B and Sharma B, Transdermal patch: A discrete dosage form. International Journal of Current Pharma Research, 3, 2011, 98-108.

[2] Sharma N, Agarwal G, Rana AC, Bhat Z and Kumar D, A Review: Transdermal drug delivery system: A tool for novel drug delivery system. International Journal of Drug Development and Research, 3, 2011, 70-84.

[3] Arti K, Ajit KY, Sunil S, Henendra G, Haribansh NS, Anamika S, Theoretical aspects of transdermal drug delivery system, Bulletin of Pharmaceutical Research, 3(2), 2013, 78-89.

[4] Jalwal P, Jangra A, Dhaiya L, Sangwan Y, Saroha R, A review on transdermal patches. Pharmaceutical Research, 3, 2010, 139-149.

[5] Rastogi V, Yadav P, Transdermal drug delivery system: An overview, Asian Journal of Pharmaceutics 6(3), 2012, 161-170

[6] Kapoor D, Patel M and Singhal M, Innovations in Transdermal drug delivery system, International Pharmaceutica Sciencia, 1(1), 2011, 54-61
[7] Keleb E, Sharma, RK, Mosa Esmaeil B and Abdalkadar $\mathrm{Z}$ aljahwi, Review on Transdermal Drug Delivery SystemDesign and Evaluation, International Journal of Advances in Pharmaceutical Sciences, 1, 2010, 201-211.

[8] Patel D, Sunita A, Parmar B, Bhura N, Transdermal Drug Delivery System: A Review, The Pharma Innovation 1(4), 2012, 66-75.

[9] Nikhil S, Geta A, Rana AC, A Review Transdermal Drug Delivery System a tool for Novel Drug Delivery System, International Journal of Drug Development and Research, 3(3), 2011, 70 .

[10]Rohit T, Manish J, Shailender M, Sanjay S, Transdermal Drug Delivery System: A Review. International Journal of Therapeutic Applications, 14, 2013, 2228.

[11]Chandrashekhar NS, Shobha Rani RH. Physicochemical and Pharmacokinetic Parameters in Drug Selection and Loading of Transdermal Drug Delivery, Indian Journal of Pharmaceutical Sciences, 70(1), 2008, 94-96.

[12]Vinod KR, Sarvani P, Banji D and Teja BB, Transdermal drug delivery systemover coming challenges of popular drug delivery system. 
International Journal of Pharma World Research 1, 2010, 1-14.

[13] Bhargava $T, \quad$ Ramchandani $U$, Shrivastava SK and Dubey PK, Current trends in NDDS with special reference to NSAIDs. International Journal of Pharmacy and Bio Sciences, 2, 2011, 92114.

[14]D Bhowmik, PK Rao, S Duraivel and Sampath Kumar, Recent Approaches In Transdermal Drug Delivery System, The Pharma Innovation, 2(3), 2013, 99-108.

[15]Alexander, S Dwivedi, Ajazuddin, TK Giri, S Saraf, S Saraf, and DK Tripathi, Approaches for Breaking the Barriers of Drug Permeation Through Transdermal Drug Delivery, Journal of Controlled Release, 164(1), 2012, 26-40.

[16]Dhiman S, Thakur GS, Rehni AK. Transdermal patches: a recent approach to new drug delivery system, International

Journal of Pharmacy and Pharmaceutical Sciences, 3(5), 2011, 26-34.

[17] Shalu Rani, Kamal Saroha and Navneet Syan, Transdermal Patches a successful tool in Transdermal Drug Delivery System: An overview, Der Pharmacia Sinica, 2(5), 2011, 17-29.

[18] Eleb E., Sharma, RK, Mosa Esmaeil, B, Abdalkadar $\mathrm{Z}$ aljahwi, Review on
Transdermal Drug Delivery SystemDesign and Evaluation, International Journal of Advances in Pharmaceutical Sciences, 1, 2010, 201-211.

[19] Singh MC, Naik AS and Sawant SD, Transdermal Drug Delivery Systems with major emphasis on Transdermal patches: A Review. Journal of Pharmacy Research, 3(10), 2010, 2537-2543.

[20] Archana KG, Transdermal drug delivery system: Formulation aspects and evaluation, Journal of Pharmaceutical Sciences, 1(1), 2013,1-10.

[21]Sugibayashi $\mathrm{K}$ and Morimoto $\mathrm{Y}$, Polymers for Transdermal Drug Delivery System, Journal of Controlled Release, 29, 1994, 177-185.

[22] Shingade GM, Aamer Q, Sabale PM, Grampurohit ND, Gadhave MV, Review on: recent trend on transdermal drug delivery system, Journal of Drug Delivery \& Therapeutics, 2(1), 2012, 6675.

[23] Mathur V, Satrawala Y and Rajput MS, Physical and chemical penetration enhancers in transdermal drug delivery system, Asian Journal of Pharmacy 4(3), 2010, 173-183.

[24]Panner Selvam, Anoop Kumar Singh, Sivakumar T, Transdermal drug delivery systems for antihypertensive drugs - A 
review, Indian Journal of Pharmaceutical and Biological Research, 1(1), 2010, 1-8.

[25] Divyesh Patel, Nirav Patel, Transdermal

Drug Delivery System Review, International Journal of Biopharmaceutical and Toxicological Research, 1(1), 2011, 61-80.

[26] Patel D, Patel N, Parmar M and Kaur N, Transdermal drug delivery system: Review. International Journal of Biopharm and Toxicological Research, 1, 2011, 61- 80.

[27]Eseldin Keleb, Rakesh Kumar Sharma, Transdermal Drug Delivery SystemDesign and Evaluation, International Journal of Advances in Pharmaceutical Sciences, 1(3), 2010, 201-211.

[28] Patel RP and Baria AH, Formulation and evaluation considerations of transdermal drug delivery system, International Journal of Pharmaceutical Research, 3, 2011, 1-9.

[29] Sakalle P, Dwivedi S and Dwivedi A, Design, Evaluation, Parameters and Marketed Products of transdermal patches: A Review. Journal of Pharmacy Research, 3(2), 2010, 235-240. 